\title{
Iatrogenic Cushing's syndrome due to drug interaction between glucocorticoids and the ritonavir or cobicistat containing HIV therapies
}

\author{
Authors: Emilie R Elliot, ${ }^{\mathrm{A}}$ Aikaterini Theodoraki, ${ }^{\mathrm{B}}$ Lakshmi R Jain, ${ }^{\mathrm{C}}$ Neal J Marshall, ${ }^{\mathrm{D}}$ Marta Boffito, ${ }^{\mathrm{E}}$ \\ Stephanie E Baldeweg ${ }^{F}$ and Laura J Waters ${ }^{G}$
}

Ritonavir and cobicistat, used as pharmacokinetic enhancers in combination with some antiretrovirals (ARVs) for the treatment of HIV, are potent inhibitors of the CYP3A4 isoenzyme. Most glucocorticoids are metabolised via the CYP3A4 pathway and iatrogenic Cushing's syndrome (ICS), with possible secondary adrenal insufficiency (SAI), is a recognised complication following co-administration with ritonavir or cobicistat. A structured approach for identifying and managing potentially affected individuals has not been established.

We systematically identified patients with ICS/SAI and found substantial heterogeneity in clinical practice across three large London HIV centres. While this significant drug interaction and its complications are now well-recognised, it is apparent that there is no standardised approach to management or guidance for the general physician. Here we describe the management of ICS/SAI in our current practice, review the available evidence and suggest practice recommendations.

KEYWORDS: Adrenal insufficiency, cobicistat, Cushing's syndrome, HIV, ritonavir

\section{Introduction}

Ritonavir and cobicistat are pharmacokinetic enhancers used in the treatment of HIV infection. They are extremely potent inhibitors of cytochrome P450 3A4 (CYP3A4) activity. Ritonavir, originally developed as an antiretroviral, is used

Authors: ${ }^{\text {A }}$ specialty registrar in HIV/genito-urinary medicine, Chelsea and Westminster Hospital, London, UK; ${ }^{\text {B }}$ specialty registrar in endocrinology, University College London Hospitals Foundation Trust, London, UK; ${ }^{C}$ specialty registrar in GUM/HIV, Mortimer Market Centre, London, UK; ${ }^{D}$ specialty pharmacist in HIV, Ian Charleson Centre for HIV Medicine, Royal Free London NHS Foundation Trust, London, UK; ${ }^{E} H I V$ consultant physician, Chelsea and Westminster Hospital, London, UK; F consultant physician in diabetes and endocrinology, University College London Hospitals Foundation Trust, London, UK; ' HIV consultant physician, Mortimer Market Centre, London, UK at sub-therapeutic doses, in combination with HIV protease inhibitors (PIs), to significantly increase their concentrations and allow less frequent and lower dosing. ${ }^{1}$ Cobicistat was developed more recently and is similar in structure. It is used in combination with PIs or elvitegravir (an integrase inhibitor) ${ }^{2}$ but has no antiretroviral activity itself. ${ }^{3}$

The downside of this pharmacokinetic manipulation is the significant potential for interactions with CYP3A4 substrates, leading to side effects. CYP3A4 is the dominant isoenzyme of the hepatic cytochrome $\mathrm{P} 450$ system and is the primary metabolic step for the degradation of endogenous and most prescribed corticosteroids. The metabolism of these can therefore be decreased by inhibitors such as ritonavir/cobicistat. ${ }^{4}$ Subsequent increases in exogenous corticosteroid plasma concentrations and half-life can lead to iatrogenic Cushing's syndrome (ICS $)^{5}$ and, at supraphysiological levels, to suppression of adrenocorticotropic hormone (ACTH) and endogenous corticosteroid secretion, potentially resulting in secondary adrenal insufficiency (SAI). There is abundant evidence highlighting this issue. ${ }^{1,6-11}$ However, a structured approach to identify and manage potentially affected individuals has not been established. We aim to summarise the current management of ICS/SAI in three large HIV patient cohorts, review the available literature and develop practice recommendations. Of note, cobicistat became available after the evaluation period covered; however, all advice for ritonavir is applicable to it. ${ }^{12}$

\section{Methods}

\section{Service evaluation}

This evaluation was carried out at three centres: Royal Free Hospital, London (A), Mortimer Market Centre, University College Hospital, London (B) and Chelsea and Westminster Hospital, London (C). Patients with ICS/SAI were systematically identified through a search of clinic letter databases (centres A and $\mathrm{B}$ ), pathology results (centres B and C) and/or radiology interventions (centre A) at these three London HIV tertiary centres over a 9-month period. For the letter database search, search terms cortisol, budesonide, triamcinolone and fluticasone were used. For the pathology search, all cortisol and short synacthen test (SST) results for patients with HIV in the 
evaluation period were reviewed. Centre A also evaluated all radiological interventions in HIV patients during the study period. Not all search modes were available in all three centres. For instance, some software used does not permit mass scanning of individual clinic letter content and not all pathology/ radiology departments had the resources to release data.

Case notes were then reviewed retrospectively for all identified cases. Presenting features, steroid indication, formulation, doses and route, concurrent antiretrovirals (ARVs), investigations and management were recorded.

As a service evaluation, ethical approval was not required in accordance with NHS Health Resource Authority guidance.

\section{Literature review}

The available literature was reviewed using the bibliographic database PubMed and the following search terms: iatrogenic Cushing's/Cushing syndrome, secondary adrenal insufficiency, ritonavir, corticosteroids, glucocorticoids, fluticasone, budesonide, mometasone, triamcinolone, methylprednisolone, prednisolone, CYP3A4 inhibitors and HIV.

\section{Results}

\section{Service evaluation}

The service evaluation ran from 31 January 2012 to 1 October 2012. From a combined cohort of approximately 15,000 patients, 19 cases of confirmed ARV-steroid interactions leading to ICS/ SAI were identified over 9 months (three in centre A, seven in centre $\mathrm{B}$ and nine in centre $\mathrm{C}$ ). Cases are described in detail in Table S1; 13 out 19 cases were male and ages ranged from 36 to 64 years. Overall, the main indications for steroid use were asthma/chronic obstructive pulmonary disease (COPD) $(n=8)$ and musculoskeletal $(n=6)$. Twelve patients presented with typical features of Cushing's syndrome and seven with symptoms consistent with adrenal insufficiency. Fluticasone (inhaled or nasal) was the culprit steroid in over a half of cases $(n=10)$. There was one case of topical steroid use.

Large intercentre differences in investigations and management were seen: 14 out of 19 patients had a 9 am cortisol measured in their HIV clinic, with evidence of adrenal suppression seen in most $(n=12)$. Meanwhile, only 10 patients had a documented SST, all with confirmed SAI. Two patients had investigations carried out by their GPs, but seven did not have an SST (mainly in centre $C, n=5$ ). For these patients, clinical findings and/or the 9 am cortisol (range: 11-301 nmol/L) were cited as diagnostic.

With regards to pharmacological management, 58\% ( $\mathrm{n}=11)$ switched or stopped the steroids alone, $21 \%(n=4)$ switched ARV alone and $21 \%(n=4)$ did both. Centre B ceased use of the steroid in all of its patients, unlike A and C. Centre A switched ARVs in all of its patients (while continuing steroids in two of the three patients), whereas centre B switched ARVs in two of nine patients and $\mathrm{C}$ in three of seven, seemingly unrelated to whether the steroid had been injected.

Over half of patients $(n=11)$ received hydrocortisone $(\mathrm{HC})$ replacement, the majority of which were in centres $\mathrm{A}(3 / 3)$ and B (6/9), while centre C prescribed HC in only two patients, even when background steroids had been stopped. Initial HC replacement dose varied from a total daily dose of $20 \mathrm{mg}$ in three divided doses $(10 / 5 / 5 \mathrm{mg})$ to just $10 \mathrm{mg}$ once daily. The duration of treatment ranged from 2 to 72 weeks.

Overall, ten patients were co-managed with the endocrinology team. Centre A co-managed all of its cases, while centre B comanaged five patients and centre $\mathrm{C}$ two. Advice on emergency steroid cover was documented in only eight cases (38\%) and only one case was reported as a serious untoward incident.

Nine patients (48\%) had documented monitoring for hypothalamic-pituitary-adrenal (HPA) axis recovery (mainly centres A and B). Documented recovery time ranged from 12 weeks to not recovered at 3.5 years. Correspondence to primary care physicians (GPs) was sent for all except three patients; these three patients did not wish to disclose their HIV status.

\section{Literature review}

The literature was reviewed up to January 2016 and referenced search results range from 1994 to 2015.

\section{Features and timing of ICS and SAI}

The manifestations of ICS and adrenal insufficiency are summarised in Table 1. Some manifestations of glucocorticoid excess occur quickly (hours/days), for example psychiatric effects and increased appetite, whereas a cushingoid appearance, glucose intolerance or osteoporosis may take longer to develop (weeks/months). ${ }^{13}$ In our cohort, changes in appearance (weight gain, striae, proximal myopathy) and fatigue were the commonest presenting features; however, presentations can vary significantly. ${ }^{6-11}$

Although glucocorticoid treatment courses of less than 3 weeks duration often will not lead to HPA axis suppression, at relatively high doses, significant HPA suppression can occur in as little as 5 days. ${ }^{13,14}$ Longer-acting glucocorticoid formulations are associated with a higher risk of SAI. ${ }^{15,16}$

\begin{tabular}{ll} 
Table 1. Signs and symptoms of iatrogenic \\
Cushing's syndrome and secondary adrenal \\
insufficiency & \\
\hline Features of iatrogenic & Features of secondary \\
Cushing's syndrome & adrenal insufficiency \\
$>$ Weight gain - central & $>$ Weakness/fatigue \\
obesity with redistribution & $>$ Malaise \\
of body fat to truncal & $>$ Nausea \\
areas, dorsocervical and & $>$ Vomiting \\
supraclavicular fat pads & $>$ Abdominal pain \\
$>$ Moon face & $>$ Anorexia/weight loss \\
$>$ Plethora & $>$ Myalgia \\
$>$ Easy bruising & $>$ Arthralgia \\
$>$ Thin skin & $>$ Hypotension \\
$>$ Dark abdominal striae & $>$ Lethargy \\
$>$ Proximal myopathy & $>$ Unexplained hypogly- \\
$>$ Susceptibility to infections & caemia \\
$>$ Depression and psychosis & $>$ Hyponatremia \\
$>$ Avascular necrosis & $>$ Seizure \\
$>$ Osteoporosis - trabecular &
\end{tabular}




\section{Current evidence on individual glucocorticoids by route}

\section{Inhaled}

Fluticasone causes more cortisol suppression than other inhaled steroids (including beclomethasone, budesonide, triamcinolone and flunisolide) because of its longer half-life and its higher binding affinity for the glucocorticoid receptor. ${ }^{17}$ Manufacturers of fluticasone specifically warn of co-administration with ritonavir. ${ }^{18}$ Drug interactions are also well described between budenoside and ritonavir. ${ }^{6,7,19}$ Conversely, there are no reported cases with beclomethasone interaction as it is predominantly metabolised by esterase hydrolysis; ${ }^{20}$ CYP3A4 is minimally involved. In a randomised healthy volunteer study, the addition of ritonavir to inhaled beclomethasone doubled the area under the curve (AUC) for the serum concentration of the active metabolite of beclomethasone, but with no significant reduction in basal or stimulated serum cortisol levels. ${ }^{20}$

\section{Nasal}

SAI secondary to nasal fluticasone is well documented, whereas beclomethasone is not predicted to cause cortisol suppression. ${ }^{12,18}$ A few cases on mometasone have been published but with unclear contributory effect on symptoms. ${ }^{21,22}$ Therefore, while not thought to cause more cortisol suppression than beclomethasone, ${ }^{23}$ mometasone is metabolised via CYP3A4 and caution is advised.

\section{Intra-articular or epidural}

ICS/SAI have been observed with intra-articular triamcinolone and methylprednisolone administered without pharmacological boosting. ${ }^{24,25}$ In HIV, there are at least 30 published cases of ICS/SAI following intra-articular/epidural steroid in patients on antiretrovirals; ${ }^{8-11,26} 26$ were on ritonavir and 4 on other ARVs. All of these patients were injected with triamcinolone, except one who was injected with methylprednisolone. ${ }^{8-11,26-30} \mathrm{~A}$ retrospective review reported an $11 \%$ event frequency, with the biggest risk factor being two or more injections within 6 months. ${ }^{26}$ Interestingly, a recent randomised controlled trial demonstrated no efficacy advantage of triamcinolone over methylprednisolone for knee osteoarthritis (in a non-HIV population). ${ }^{31}$ Methylprednisolone is metabolised by the CYP3A4 enzyme and ICS/SAI following co-administration with a pharmacological CYP3A4 inhibitor is theoretically possible. ${ }^{32}$ However, in view of strong evidence of ICS/SAI with triamcinolone, no efficacy benefit and only one case reported with methylprednisolone, the latter may be preferable in patients on ritonavir/cobicistat.

\section{Topical and ocular}

ICS/SAI are widely reported with the use of potent topical steroids over a wide skin surface area in patients without HIV. ${ }^{33}$ As such, the risk of systemic absorption and pharmacological boosting of topical steroids in the context of ritonavir or cobicistat is real and warrants close monitoring. Two cases of low morning cortisol/ACTH following concomitant use of ritonavir and topical triamcinolone have been reported at a conference, but none in the published literature. ${ }^{21}$ Outside the use of CYP3A4 inhibitors, ICS has only been reported on a few occasions with ocular steroid drops ${ }^{34}$ and in two cases in the context of ritonavir. ${ }^{35,36}$
Oral

A $30-40 \%$ increase in AUC has been reported in healthy volunteers taking prednisolone with ritonavir, ${ }^{37}$ and there is one case of ICS with the use of oral budesonide and ritonavir. ${ }^{19}$

\section{Discussion}

Using a systematic approach, our review identified 19 cases of significant drug-drug interactions between ARVs and steroids over 9 months across three large HIV centres. There were substantial inter-centre discrepancies in investigations and management.

Not all patients with clinical signs had a 9 am cortisol carried out and just over half underwent dynamic testing (depending on the centre). For those who did not have an SST, clinical findings and the 9 am cortisol were used. Interestingly, three patients did not even meet the cortisol cut offs recently suggested by Tomlinson et al as sufficient to justify avoiding an SST $(<35$ and $>348 \mathrm{nmol} / \mathrm{L}$ in patients taking inhaled glucocorticoids). ${ }^{14}$

Just over half of patients were co-managed with the endocrine team; this was unevenly distributed across the centres and may reflect the availability of local referral pathways. Pharmacological management was also very varied, with some clinicians stopping/switching the steroid alone, others switching the ARVs and stopping the steroid and some opting to switch ARVs only. There was no clear relationship with the steroid method of administration (eg injected). While this will be influenced in parts by the patient's clinical situation and viral resistance profile, it may also reflect a lack of consensus in clinical priority among practitioners (ARVs versus steroid). Communication with GPs was high across all centres.

These findings highlight a lack of a standardised approach to practice, possibly reflecting the lack of published guidance available. Here, we suggest practice recommendations for the prevention, investigation and management of drug interactions between steroids and pharmacologically enhanced antiretrovirals, with the aim of providing guidance and reducing heterogeneity in practice.

\section{Prevention of pharmacological interaction via safe prescribing in patients treated with CYP3A4 inhibitors}

Routine use of potent steroids metabolised via CYP3A4, including fluticasone, budesonide and triamcinolone in patients treated with CYP3A4 inhibitors is not recommended via any route. If unavoidable, the HIV team should review the possibility of a switch to a non-ritonavir/cobicistat containing regimen, carefully considering known or likely resistance to HIV drugs. This should ideally precede the introduction of the steroid. If co-administration of ritonavir/cobicistat with a highrisk steroid absolutely cannot be prevented, clear interspecialty (including GP) communication and close monitoring are critical. A risk-benefit discussion with the patient is important.

\section{Recommendations for inhaled/intranasal steroids}

The preferred alternative to fluticasone and budesonide, via inhaled or nasal route, is beclomethasone. The use of steroid sparing interventions should be considered in conjunction with the appropriate specialty team. 
Some steroid preparations may be used safely.

Examples of these include:

- Hydrocortisone cream or ointment

- Inhalers or allergy nasal sprays containing beclomethasone (eg Qvar ${ }^{\circledR}$, Becotide ${ }^{\circledR}$, Beconase ${ }^{\circledR}$ or Fostair(®)

For further information please ask your clinic doctor or pharmacist. Alternatively you can contact Medicines Information

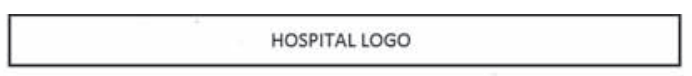

This card contains important information about one of the medications you have been prescribed.

Please read it carefully and show it to any healthcare professionals who may be providing treatment for you.

It is a good idea to carry this card with you at all times.
You have been prescribed a medication (protease inhibitor, cobicistat or ritonavir) that interacts with some steroid preparations.

These preparations include inhalers, joint injections (eg for tennis elbow), nose or eye drops/sprays, tablets and some creams/ointments.

Taking these preparations can result in serious complications as a result of producing very high steroid levels in the body.
Some steroid preparations are prescribed and others can be purchased over the counter from a pharmacy. If you are prescribed, administered or buy a steroid preparation please check that they do not contain the following

Fluticasone - contained in Seretide (4), Flixotide(a), Flutiform (i) and Relvar Ellipta(i) inhalers and in some 'allergy' nasal preparations (eg Flixonase or Boots allergy relief spray)

Triamcinolone - contained in some joint injections such as Adcortyla or Kenalogis

Budesonide - contained in some inhalers (eg Pulmicort or Symbicort

Mometasone - contained in some inhalers (eg Asmanex (i)) and nasal sprays (eg Nasonex 8 )

Dexamethasone - contained in some tablets (eg Dexsole)

and eye drops (eg Maxidex (a) or Maxitrol (i))

Fig 1. Patient information leaflet on potential drug interactions between glucocorticoids and antiretroviral boosters.

\section{Recommendations for injectable steroids}

Triamcinolone is not recommended in patients on ritonavir/ cobicistat. Intra-articular methylprednisolone may be used as an alternative, although the potential for ICS and SAI still exists. Some centres advise a dose reduction of $30 \%$ for methylprednisolone; however, this is guided by expert opinion only. Non-steroid options in this setting are preferable.

\section{Recommendations for oral steroids}

Duration of use of oral steroids should be kept to a minimum, depending on the initial indication, with a switch to a steroidsparing regimen as soon as possible. Consideration should be given to dose modification and potential for steroid taper, although there is little evidence to guide this.

\section{Recommendations for topical steroids}

The prolonged use of topical corticosteroids (depending on the steroid potency, skin condition, surface body area and involvement of face, groin, and/or axillary regions) with CYP3A4 inhibitors poses a risk of CSI/SAI. ${ }^{22,33}$ Steroid sparing alternatives should be sought with dermatology input.

\section{Prevention of pharmacological interaction via integration and patient empowerment}

In the UK, ARVs are prescribed by HIV clinicians and dispensed via hospital pharmacies or home delivery. NonHIV medications, such as steroids, are usually prescribed by non-HIV clinicians and dispensed locally or bought over the counter. Consequently, potential interactions that would be detected if drugs were prescribed or dispensed at the same site may go unnoticed. Although there have been national efforts to communicate HIV drug interaction information to GPs, treatment decisions (eg triamcinolone injections) may occur in other clinical settings and interactions may be missed. Additionally, patients with HIV may not disclose their status to other treating physicians. In light of this, two centres have produced a patient information leaflet about potential interactions with antiretroviral boosters (Fig 1).

\section{Recommendations}

$>$ All clinicians should assess the potential for drug-drug interactions with prescribed medicines, including steroids, and seek advice from the HIV specialist team where identified. Multiple sources are available, eg Liverpool HIV drug interactions website (www.hiv-druginteractions.org), prescribing interaction checkers or the British National Formulary. Advice may vary between sources. ${ }^{38}$

$>$ A general warning for potential drug interactions with all medicines and resources should be included in all clinic letters.

$>$ Appropriate incident reporting, feedback and education will contribute to incident prevention.

$>$ Patients should be advised to check for drug interactions with all new medicines. Patient information and education may help prevent harmful interactions.

\section{Investigation of ICS/SAI and management of high- risk co-prescription}

Once a potential drug interaction has been identified, the excessive exogenous steroid needs to be discontinued and the 


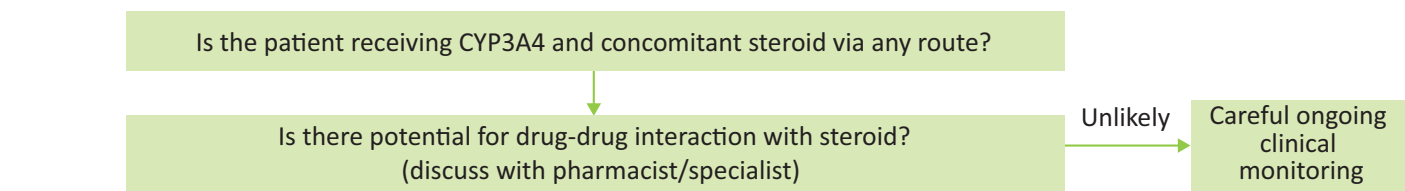
(discuss with pharmacist/specialist)

Inhaled/nasal/oral/topical steroids

\section{Inhaled/nasaloraltopical steroids}

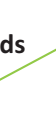

\begin{abstract}
CYP3A4 inhibitor where possible.

If patient acutely unwell,
hypotensive \pm hyponatraemic:
measure serum cortisol, commence
steroid replacement and discuss with
endocrine team
\end{abstract}

Conduct joint review of the steroid first with specialist/endocrine/HIV teams: - inhaled steroids - switch to beclomethasone or a steroid-sparing agent - oral steroids - minimise dose and duration, consider switch to steroid sparing agents - topical steroids - limit exposure, consider switch to steroid sparing agents. If steroid with high interaction risk is unavoidable, discuss with HIV team to remove

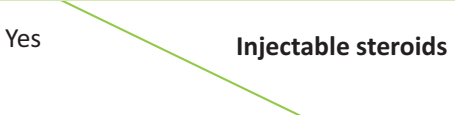

\section{le steroids}

IM/IA/epidural:

- This cannot be removed so discuss with HIV to switch CYP3A4 inhibitor.

- If not possible, monitor closely.

- Avoid further injections - consider DMARDs in future.
If 9 am cortisol $<450 \mathrm{nmol} / \mathrm{L}^{*}$, arrange for SST

\begin{tabular}{l|l|l}
$\begin{array}{l}\text { If SST normal (peak } \\
\text { cortisol }>550 \mathrm{nmol} / \mathrm{L}^{*} \text { ), } \\
\text { no need for further } \\
\text { testing. Monitor } \\
\text { closely }\end{array}$ & $\begin{array}{l}\text { If abnormal SST (peak cortisol }<550 \mathrm{nmol} / \mathrm{L}^{*} \text { ), } \\
\text { start steroid replacement in patients with } \\
\text { symptoms of adrenal insufficiency } \\
\text { In asymptomatic patients, discuss with local } \\
\text { endocrine team }\end{array}$ \\
$\begin{array}{ll}\text { If ongoing exposure to } \\
\text { CYP3A4 inhibitor and } \\
\text { steroid, return to top } \\
\text { of chart and follow }\end{array}$ & $\begin{array}{l}\text { Repeat the morning cortisol and/or the } \\
\text { SST every } 8 \text { weeks in order to assess for } \\
\text { recovery of the HPA axis }\end{array}$ \\
& $\begin{array}{l}\text { Stop any steroid replacement once } 9 \text { am } \\
\text { cortisol } \geq 450 \mathrm{nmol} / \mathrm{L}^{*} \text { or post SST cortisol }\end{array}$ \\
\hline
\end{tabular}

If 9 am cortisol $\geq 450 \mathrm{nmol} / \mathrm{L}^{*}$, monitor clinically No need for further testing unless very high index of suspicion of SAI

If ongoing exposure to CYP3A4 inhibitor and steroid, return to top of chart and follow

Other steps:
- patients with SAI - provide information leaflet with
steroid sickness rules and advise to attend A\&E if
they become unwell
- complete incident form and inform patient's GP
and other relevant specialists.

Fig 2. Investigation and management pathway for patients with potential or suspected glucocorticoid-CYP3A4 inhibitor interaction (expert opinion). *Depending on local assay. A\&E = accident and emergency department; DMARD = disease-modifying antirheumatic drugs; HPA = hypothalamic-pituitaryadrenal; IA = intra-articular; IM = intra-muscular; SAI = secondary adrenal insuffi ciency; SST = short synacthen test.

patient investigated for ICS/SAI (Fig 2), where the most striking biochemical finding is a suppressed endogenous serum cortisol. While ICS is a clinical diagnosis, a 9 am cortisol is useful as a screening test for consequent SAI. ${ }^{14}$ A 9 am cortisol of less than $100 \mathrm{nmol} / \mathrm{L}$ usually indicates deficient basal secretion or the presence of a potent exogenous steroid. A cortisol of greater than $550 \mathrm{nmol} / \mathrm{L}$ shows an intact HPA axis. ${ }^{13}$ Of note, many use a lower threshold of greater than $450 \mathrm{nmol} / \mathrm{L}$ and greater than $348 \mathrm{nmol} / \mathrm{L}$ has been suggested in those on current inhaled glucocorticoids. ${ }^{14,39}$ Nevertheless, patients who have 9 am cortisol levels of less than $450 \mathrm{nmol} / \mathrm{L}$ should be further investigated. The standard SST is the most frequently used test in this context ${ }^{40}$ where 0 minute, 30 minute and 60 minute cortisol levels are measured after a $250 \mu \mathrm{g}$ tetracosactide injection. Cortisol thresholds and assays may vary and should be checked with the local laboratory. Plasma ACTH is also low but this test is not routinely used. ${ }^{39}$

Concomitant synthetic steroid use can interfere with cortisol measurements. Screening should therefore be timed, if possible, at least 1 week after the exogenous glucocorticoid has been tapered to a physiological dose or removed. ${ }^{13}$ Prolonged released formulations (intra-articular and epidural, especially triamcinolone), cause low level cortisol and a blunted response to ACTH stimulation for 4-6 weeks, outside of CYP3A4 inhibition ${ }^{41,42}$ and likely for longer with it. Test results need to be interpreted with caution and with endocrinology input. In asymptomatic patients, it may be prudent to delay testing for 4-6 weeks post steroid injection.

\section{Recommendations}

> A 9 am cortisol level is helpful to rule out SAI ( $>450 \mathrm{nmol} / \mathrm{L}$, bearing in mind local variation).

$>$ For 9 am cortisol $<450 \mathrm{nmol} / \mathrm{L}$, an SST will distinguish patients with intact HPA axis from those with SAI. This test is not suitable for patients on current supraphysiological exogenous steroids and should be organised and interpreted with the endocrine team. 
$>$ Exogenous steroid cessation is dependent on the route of administration:

- inhaled/intranasal - switch to beclomethasone or steroidsparing agent

- oral - ensure only physiological (replacement) dose is administered

- topical - discuss with dermatology for steroid sparing agents

- injected - stop further administrations, consider diseasemodifying agents.

In asymptomatic patients, it is recommended to stop/switch the potent steroid prior to investigations. The patient should be warned of potential symptoms of adrenal insufficiency and advised to seek urgent medical attention for steroids and investigation if these develop. An information leaflet can be provided. Some specialists opt to prescribe empirical hydrocortisone cover prior to investigations. Local endocrinology team input is recommended. If steroid cessation is clinically impossible, discuss with the HIV team to consider switching ARVs. Do not cease steroids and ritonavir/cobicistat at the same time, without steroid replacement cover or evidence of an intact HPA axis.

In symptomatic patients with suspected SAI, start hydrocortisone $20 \mathrm{mg} /$ day in 2-3 divided doses before planning investigations. If the patient is acutely unwell, hypotensive and/or hyponatraemic, measure serum cortisol, commence stress dose steroid replacement therapy and discuss with the endocrine team.

On the day of cortisol/SST testing, hydrocortisone is withheld until the test has ended, as is the previous evening's dose.

In patients with confirmed SAI, repeat 9 am cortisol/SST at regular intervals (for example every 8 weeks) to identify and document HPA axis recovery.

\section{Treatment of patients with symptomatic SAI}

Symptomatic patients with SAI should be treated with daily replacement glucocorticoids doses plus 'stress doses' during physiological stress (illness, injury or surgery). ${ }^{13,16}$ Physiological glucocorticoid doses are equivalent to prednisolone

$5.0-7.5 \mathrm{mg}$ /day, or hydrocortisone $20 \mathrm{mg}$ /day (in 2-3 doses). Because of the short half-life of hydrocortisone, there may be more opportunity for the HPA axis to recover compared with longer acting steroids. ${ }^{13}$ Stress dose steroids usually involve doubling the oral glucocorticoid dose, or the use of parenteral steroids in patients unable to have oral steroids. ${ }^{16}$ An individual with biochemical evidence of SAI in the absence of symptoms is also at risk of adrenal crisis during physiological stress and should receive 'stress doses', with or without daily physiological glucocorticoids. There is no evidence that treating asymptomatic patients with SAI delays the recovery of the HPA axis.

\section{Recommendations}

> Treat all symptomatic patients with SAI with oral replacement glucocorticoids doses, such as hydrocortisone $20 \mathrm{mg} /$ day in 2-3 divided doses, typically $10 \mathrm{mg}$ on waking, $5 \mathrm{mg}$ at 12:00 and $5 \mathrm{mg}$ at 16:00, or prednisolone $5.0-7.5 \mathrm{mg} /$ day in a single morning dose.

$>$ Provide all patients with SAI with written advice including stress doses of steroids (eg www.addisons.org.uk/publications).

> Manage care jointly with the HIV and endocrine teams.

\section{Limitations}

The methods adopted in this service evaluation only identified symptomatic and diagnosed patients and is likely to have underestimated the actual incidence of ICS/SAI. Data from patients' records did not allow accurate assessment of the duration of steroid therapy and we were not able to analyse data from patients who were investigated by their GP.

\section{Conclusion}

Using a systematic approach, we identified patients with ICS and SAI following co-prescription of ARVs and steroids and found significant variability in clinical practice across three large London centres. The key to prevention is prospective identification and avoidance of the harmful drug interaction through communication and increased awareness among clinicians and patients with HIV. We have produced a relevant patient information leaflet and clear joint recommendations in order to manage these patients safely.

\section{Conflicts of interests}

ERE has received speaking and travel grants from Janssen, ViiV, Bristol-Myers Squibb, Merck Sharp \& Dohme, and Gilead. NJM has received honoraria Gilead, Abbvie, Janssen, MSD, BMS, ViiV, Mylan and Accord for acting as a speaker or advisor. $\mathrm{MB}$ has received honoraria for being a speaker or advisor and clinical research grants from Gilead, Janssen, MSD, BMS, ViiV, Cipla, Teva and Mylan. LJW has received conference support, speaker fees, advisory fees or research grants from Gilead, MSD, BMS, AbbVie, ViiV and Janssen. AT, LRJ and SEB have no conflicts of interest to declare.

\section{Author contributions}

ERE, AT and LRJ are joint first authors and equally analysed data, wrote the initial draft and edited subsequent versions, $\mathrm{MB}$ edited the manuscript, NJM, SEB and LJW analysed data and edited the manuscript.

\section{Supplementary material}

Additional supplementary material may be found in the online version of this article at www.clinmed.rcpjournal.org/:

S1 - Summary and management of ICS and SAI resulting from ritonavir/glucocorticoid interactions.

\section{References}

1 Samaras K, Pett S, Gowers A et al. Iatrogenic Cushing's syndrome with osteoporosis and secondary adrenal failure in human immunodeficiency virus-infected patients receiving inhaled corticosteroids and ritonavir-boosted protease inhibitors: six cases. J Clin Endocrinol Metab 2005;90:4394-8.

2 Stribild product information. London: Gilead Sciences, 2014. Available online at www.medicines.org.uk/emc/medicine/27810 [Accessed 4 August 2016].

3 Tybost product information. London: Gilead Sciences, 2014. Available online at www.medicines.org.uk/emc/medicine/28298 [Accessed 4 August 2016].

4 Daveluy A, Raignoux C, Miremont-Salame G et al. Drug interactions between inhaled corticosteroids and enzymatic inhibitors. Eur J Clin Pharmacol 2009;65:743-5. 
5 European AIDS Clinical Society. Drug-drug Interactions between Corticosteroids and ARVs. In: Guidelines Version 8.0. Brussels: EACS, 2015:23.

6 Saberi P, Phengrasamy T, Nguyen DP. Inhaled corticosteroid use in HIV-positive individuals taking protease inhibitors: a review of pharmacokinetics, case reports and clinical management. HIV Med 2013;14:519-29.

7 Kedem E, Shahar E, Hassoun G et al. Iatrogenic Cushing's syndrome due to coadministration of ritonavir and inhaled budesonide in an asthmatic human immunodeficiency virus infected patient. J Asthma 2010;47:830-1.

8 Yombi JC, Maiter D, Belkhir L et al. Iatrogenic Cushing's syndrome and secondary adrenal insufficiency after a single intra-articular administration of triamcinolone acetonide in HIV-infected patient treated with ritonavir. Clin Rheumatol 2008;27 (Suppl 2):S79-82.

9 Schwarze-Zander C, Klingmuller D, Klumper J et al. Triamcinolone and ritonavir leading to drug-induced Cushing syndrome and adrenal suppression: description of a new case and review of the literature. Infection 2013;41:1183-7.

10 Hall JJ, Hughes CA, Foisy MM et al. Iatrogenic Cushing syndrome after intra-articular triamcinolone in a patient receiving ritonavirboosted darunavir. Int J STD AIDS 2013;24:748-52.

11 Sadarangani S, Berg ML, Mauck W et al. Iatrogenic cushing syndrome secondary to ritonavir-epidural triamcinolone interaction: an illustrative case and review. Interdiscip Perspect Infect Dis 2014;2014:849432.

12 Lewis J, Turtle L, Khoo S et al. A case of iatrogenic adrenal suppression after co-administration of cobicistat and fluticasone nasal drops. AIDS 2014;28:2636-7.

13 Hopkins RL, Leinung MC. Exogenous Cushing's syndrome and glucocorticoid withdrawal. Endocrinol Metab Clin North Am 2005;34:371-84, ix.

14 Woods CP, Argese N, Chapman M et al. Adrenal suppression in patients taking inhaled glucocorticoids is highly prevalent and management can be guided by morning cortisol. Eur J Endocrinol 2015;173:633-42.

15 Henzen C, Suter A, Lerch E et al. Suppression and recovery of adrenal response after short-term, high-dose glucocorticoid treatment. Lancet 2000;355:542-5.

16 Liu D, Ahmet A, Ward L et al. A practical guide to the monitoring and management of the complications of systemic corticosteroid therapy. Allergy Asthma Clin Immunol 2013;9:30.

17 Brus R. Effects of high-dose inhaled corticosteroids on plasma cortisol concentrations in healthy adults. Arch Intern Med 1999;159:1903-8.

18 Flovent Diskus product information. Research Triangle Park, NC: GlaxoSmithKline, 2014. Available online at www.gsksource. com/pharma/content/dam/GlaxoSmithKline/US/en/Prescribing Information/Flovent_Diskus/pdf/FLOVENT-DISKUS-PI-PILCOMBINED.PDF [Accessed 4 August 2016].

19 Frankel JK, Packer CD. Cushing's syndrome due to antiretroviralbudesonide interaction. Ann Pharmacother 2011;45:823-4.

20 Boyd SD, Hadigan C, McManus M et al. Influence of low-dose ritonavir with and without darunavir on the pharmacokinetics and pharmacodynamics of inhaled beclomethasone. J Acquir Immune Defic Syndr 2013;63:355-61.

21 Bhumbra NA, Sahloff EG, Oehrtman SJ et al. Exogenous Cushing syndrome with inhaled fluticasone in a child receiving lopinavir/ ritonavir. Ann Pharmacother 2007;41:1306-9.

22 Okasaki-Gutierrez R, Poole P, Troia-Concio P, Asmuth DM. Prevalence of subclinical Iatrogenic Cushing's syndrome in patients being co-administered ritonavir and corticosteroids via inhaled, intranasal, and/or topical route [abstract]. Washington DC: AIDS Conference, 2012: abstract MOPE103.

23 Chrousos GP, Ghaly L, Shedden A et al. Effects of mometasone furoate dry powder inhaler and beclomethasone dipropionate hydrofluoroalkane and chlorofluorocarbon on the hypothalamic-pituitary-adrenal axis in asthmatic subjects. Chest 2005;128:70-7.
24 Jansen TL, Van Roon EN. Four cases of a secondary cushingoid state following local triamcinolone acetonide (Kenacort) injection. Neth J Med 2002;60:151-3.

25 Habib G, Jabbour A, Artul S et al. Intra-articular methylprednisolone acetate injection at the knee joint and the hypothalamicpituitary-adrenal axis: a randomized controlled study. Clin Rheumatol 2014;33:99-103.

26 Hyle EP, Wood BR, Backman ES et al. High frequency of hypothalamic-pituitary-adrenal axis dysfunction after local corticosteroid injection in HIV-infected patients on protease inhibitor therapy. $J$ Acquir Immune Defic Syndr 2013;63:602-8.

27 Jakeman B, Conklin J, Bouchonville $\mathrm{M}$ et al. Iatrogenic Cushing's syndrome after triamcinolone plus ritonavir-boosted atazanavir. $J$ Am Pharm Assoc 2015;55:193-7.

28 Song Y, Schroeder JR, Bush LM. Iatrogenic Cushing syndrome and secondary adrenal insufficiency related to concomitant triamcinolone and ritonavir administration: a case report and review. J Int Assoc Provid AIDS Care 2014;13:511-4.

29 John G, Ollo D, Meyer P et al. Pulmonary embolism and iatrogenic Cushing's syndrome after co-administration of injected-triamcinolone and ritonavir. AIDS 2013;27:2827-8.

30 Wood BR, Lacy JM, Johnston C et al. Adrenal insufficiency as a result of ritonavir and exogenous steroid exposure: report of 6 cases and recommendation for management. J Int Assoc Provid AIDS Care 2015;14:300-5.

31 Lomonte AB, de Morais MG, de Carvalho LO et al. Efficacy of triamcinolone hexacetonide versus methylprednisolone acetate intraarticular injections in knee osteoarthritis: a randomized, double-blinded, 24-week study. J Rheumatol 2015;42:1677-84.

32 Solu-Medrone Product Information. Sandwich, Kent: Pfizer, 2015. Available online at www.medicines.org.uk/emc/medicine/7941 [Accessed 4 August 2016].

33 Bockle BC, Jara D, Nindl W et al. Adrenal insufficiency as a result of long-term misuse of topical corticosteroids. Dermatology 2014;228:289-93.

34 Chiang MY, Sarkar M, Koppens JM et al. Exogenous Cushing's syndrome and topical ocular steroids. Eye 2006;20:725-7.

35 McConkey HZ, Williams H, Kulasegaram R et al. Orbital floor triamcinolone causing Cushing's syndrome in a patient treated with Kaletra for HIV 1. BMJ Case Rep 2013;2013:pii: bcr0220125849.

36 Molloy A, Matheson NJ, Meyer PA et al. Cushing's syndrome and adrenal axis suppression in a patient treated with ritonavir and corticosteroid eye drops. AIDS 2011;25:1337-9.

37 Penzak SR, Formentini E, Alfaro RM et al. Prednisolone pharmacokinetics in the presence and absence of ritonavir after oral prednisone administration to healthy volunteers. J Acquir Immune Defic Syndr 2005;40:573-80.

38 Marshall N, Sonecha S, Okoli C, Smith C, Boffito M. Do common medicines information resources identify drug interactions between the most frequently prescribed medicines in primary care in the UK and antiretrovirals? HIV Med 2014;15:118.

39 Endocrine Unit Imperial College Healthcare NHS Trust. Endocrinology handbook. London: Imperial College Healthcare NHS Trust, 2010:48-9.

40 Reynolds RM, Stewart PM, Seckl JR et al. Assessing the HPA axis in patients with pituitary disease: a UK survey. Clin Endocrinol 2006;64:82-5.

41 Abraham G, Demiraj F, Ungemach FR. Comparison of the hypothalamic-pituitary-adrenal axis susceptibility upon single-dose i.m. depot versus long-acting i.v. triamcinolone acetonide therapy: a direct pharmacokinetic correlation. J Endocrinol 2006;191:491-6.

42 Kay J, Findling JW, Raff H. Epidural triamcinolone suppresses the pituitary-adrenal axis in human subjects. Anesth Analg 1994;79:501-5. 